\title{
Study of Radiation Therapy Treatment Effect of Palliative Metastic Breast Cancer (PMBC) using Symptoms Improvement Ratio
}

\author{
Lara Abdelmunim I Abdelrahman*, Mohamed E Gar Elanabi and Nahla Gafer \\ College of Medical Radiological Science, Sudan university of Science and Technology, Khartoum, Sudan \\ *Corresponding author: Lara Abdelmunim I Abdelrahman, College of Medical Radiological Science, Sudan university of Science and \\ Technology, Khartoum, Sudan
}

\begin{abstract}
The study examined the association between Palliative radiotherapy and the improvement of the symptoms associated with metastatic breast cancer. These include metastatic in the spinal cord and associated pain, brain metes and associated symptoms, bone metes, Metastic in the lung and finally spread in organs such as liver and others. The study also dealt with the connection between it and the improvement of the standard of life of the patient and toxic and other important factors and was achieved through a set of international standards questionnaire by which the calibration of the result. The value of radiotherapy for palliation is well known to oncologists but not necessarily to other physicians. Using terms such as symptom improvement ratio (SIR) than traditional response rates might be more appropriate in describing the benefits of palliative radiotherapy to other health care professionals. Late detection and spread of the disease in the late stages of breast cancer due to proliferation causes multiple symptoms this study was conducted in the centre of tumours treatment in Sudan, represented in Khartoum oncology Hospital (RICK) in the period 2014 to 2017. The findings support the hypothesis that radiotherapy is an effective treatment for a proportion of patients with MBC related pain, with $35 \%$ of assessable patients experiencing a clinically meaningful improvement in their pain. Of these, $12.5 \%$ had a complete improvement in their pain. There were no specific features that differentiated the complete responders from the other $\mathrm{n}$ patients Though this may be due to the small number of complete responders. There was no association between pain response and improvement in any other symptoms, therefore, palliative radiotherapy in MBC should only be considered for pain control.
\end{abstract}

Keywords: Palliative radiotherapy; Symptoms improvement; Metastic breast cancer; Pain control

\section{Introduction}

Breast cancer is the most frequently diagnosed cancer $(23 \%$ of total cancer cases) and the leading cause of death $(14 \%$ of deaths), in females, worldwide [1]. According to the Globocan 2008 estimates, approximately 1.37 million women are diagnosed with breast cancer per year. In Sudan breast cancer is the most common case. Table 1 shows the frequency \% of the breast Cancer from January 2014 to December 2017 in radiation isotopes centre of Khartoum.

Metastatic breast cancer is also classified as Stage 4 breast cancer. The cancer has spread to other parts of the body. This usually includes the lungs, liver, bones or brain [2]. The spread of cancer usually happens through one or more of the following steps:
Table 1: Shows the frequency \% of the breast Cancer from January 2014 to December 2017 in radiation isotopes centre of Khartoum.

\begin{tabular}{|c|c|c|}
\hline Year & Number of patients & Percentage \\
\hline 2014 & 1236 & 17 \\
\hline 2015 & 1247 & 16 \\
\hline 2016 & 1229 & 15 \\
\hline 2017 & 1404 & 20 \\
\hline
\end{tabular}

a. Cancer cells invade nearby heal thy cells. When the healthy cell is taken over, it too can replicate more abnormal cells.

b. Cancer cells penetrate into the circulatory or lymph system. Cancer cells travel through the walls of nearby lymph vessels or blood vessels. 
c. Migration through circulation. Cancer cells are carried by the lymph system and the bloodstream to other parts of the body.

d. Cancer cells lodge in capillaries. Cancer cells stop moving as they are lodged in capillaries at a distant location and divide and migrate into the surrounding tissue.

e. New small Tumours grow. Can cer cells form small Tumors at the new location (called micro metastases) (Table 2).

Table 2: Stages and Classifications of breast cancer.

\begin{tabular}{|c|c|}
\hline Stage & TNM Classifications \\
\hline Stage 0: & in situ breast cancer - Tis, N0, M0 \\
\hline Stage I: & T1, N0, M0 \\
\hline Stage IIa: & T0-1, N1, M0, or T2, N0, M0 \\
\hline Stage IIb: & T2, N1, M0, or T3, N0, M0 \\
\hline Stage IIIa: & T0-2, N2, M0, or T3, N1-2, M0 \\
\hline Stage IIIb: & T4, N (any), M0, or T(any), N3, M0 \\
\hline Stage IV: & T(any), N(any), M1 \\
\hline
\end{tabular}

\section{Palliative Radio Therapy for Metastic Breast Cancer}

About $34-50 \%$ of patients receiving radiotherapy are of palliative intent (Janjan N, 2008). Similar to other clinical domains, the practice of palliative radiotherapy is always guided by basic ethical principles and available clinical evidence. It requires sophisticated assessment to balance the potential benefits and burdens to the patients with respect to patient's autonomy and expectations, and con sideration of logistical factors Palliative radiotherapy is mainly indicated to relieve various local symptoms in cancer patients; to prevent debilitation such as spinal cord compression and pathological fracture; and to achieve durable loco regional control (Wong K 2004) (Table 3). The effectiveness has been confirmed by cumulative clinical evidence.

Table 3: Indication of palliative radiotherapy.

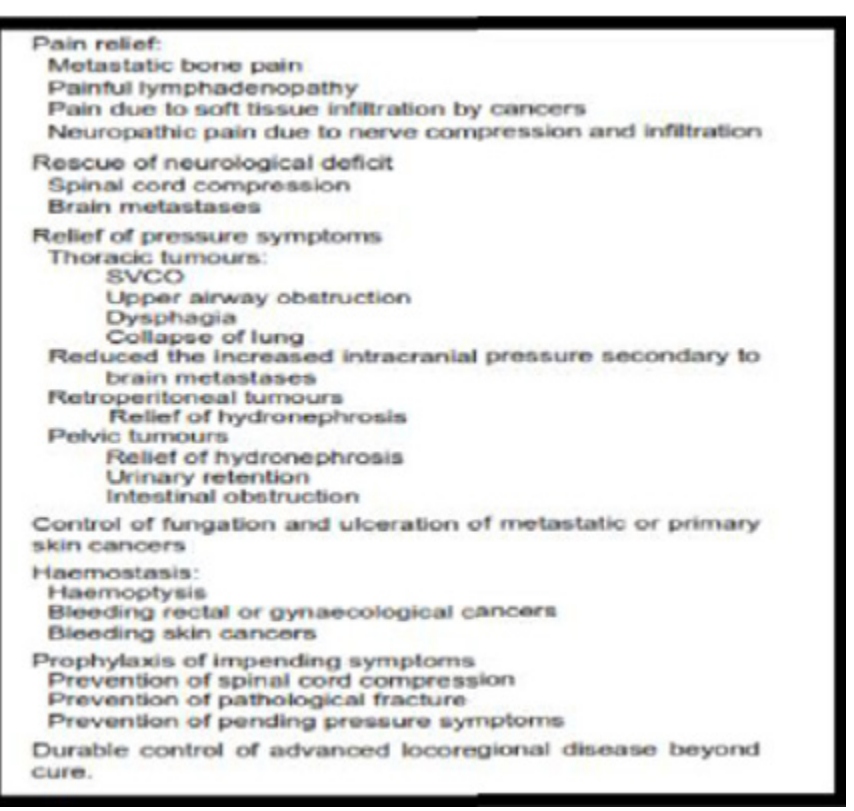

\section{Principle of Palliative Radiotherapy}

The intention of giving radiotherapy for palliation of symptoms is improvement in quality of life by decreasing or eradicating symptoms. This will not be achieved if the treatment itself induces a lot of side effects. Also, patients with metastatic cancer have a reduced life span, this may only be months, and therefore the treatment itself should not consume a major portion of the patients remaining lifespan. The major benefit of radiotherapy is the speed with which symptom improvement develops and the certainty of response. Sufficient radiation dose must be given to ensure that the symptom response will last for the rest of the patient's life. Too low a dose means retreatment at some later time is needed.

Hence guiding principles are:

1. Accurate anatomical localization of the symptomatic tumour deposit.

2. Simple treatment techniques and field arrangements.

3. Short hypo fractionated treatment regimes.

4. Moderate dose treatment to achieve a good predictable response and to keep treatment toxicity to a minimum.

5. Consider the patient's overall life expectancy when determining the treatment aims and treatment duration.

\section{Materials and Methods}

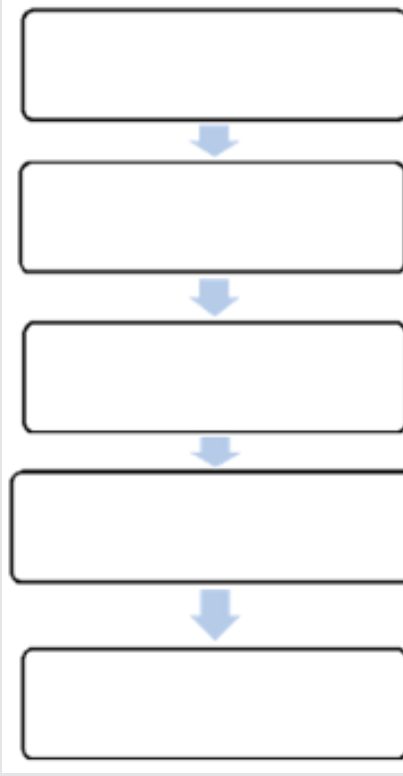

patient with metastatic breast breast cancer refereed for radiotherapy assessed for eligibility

consented and registered

week 1: (baseline) assessment undertaken

Figure 1: A study schema.

The study was conducted at Khartoum oncology hospital (RICK). The study conducted from 2014 to 2017. The rationale for this study is to development tools to assessment and evaluation patient with late stage breast cancer in symptoms control by using symptoms improvement ratio (SIR) to evaluate benefit of RT in 
palliative cases, what is mean by this is that now, in principle it is possible to evaluate the radiotherapy protocol by using simple and fast tools such as questionnaire, clinical fellow up. The patients were planning and treatment by a fluoroscopy of patient by using simple planning with two image AP/PA -LAT view of patients to detect the patient separation the images were performed using two type of machine (Hustise Castter unit, Terasix UPJ machine). The patients were planning depended on area of Metastic. Some cases were planning manual depending on previous patients' images and anatomical land mark. The patient was treating in both cobalt 60 unit or linear accelerator unit. It is analytical study where the data collected prospectively which was amongst the metastatic breast cancer patients in Sudan. Study of radiotherapy regimes for the treatment of in patients with MBC. A study schema is shown in Figure 1.

\section{Pre-Study Assessment}

One of the challenges of conducting a study examining a pain intervention in patients with advanced cancer is that changes in analgesia may occur during the study period. In such cases, it becomes very difficult to disentangle any improvements in pain as a result of the study intervention from any changes in concomitant analgesia. One of the ways this can be addressed is by stabilizing pain and analgesia prior to study entry. This approach was adopted for potential Patients in this study. Prior to study consent, patients were reviewed by Drs. Nahla gaffer and Dr Mohaga (Palliative Medicine), often with multiple visits or telephone consultations over several weeks, enabling background analgesia to be optimized and pain stabilized, where possible, before study entry. This also resulted in some patients' analgesia improving to the extent that their pain was no longer severe enough for study entry. Following optimization of analgesics, if patients were eligible, written informed consent was obtained. Baseline visit at the baseline visit an assessment of performance status was made along with a physical examination.

All previous treatments for Metastic breast cancer MBCsurgery, chemotherapy or radiotherapy - were documented along with the medication history which listed all medicines taken in the previous 24 hours. Baseline toxicity assessment was performed, and all study questionnaires were completed. In order to improve compliance, rather than leaving the patient to complete the questionnaires, a researcher completed the questionnaires based on the answers received from the patient. Take blood samples from patients at baseline and week 12 . The aims of these blood tests were twofold. The first aim was to explore the possibility that proteins could be identified which might help to predict a response to radiotherapy. The second aim was to explore the possibility of toxicity due to radiotherapy. Week 1 visit Patients were seen eight (+/-three) days after the start of radiotherapy for their week 1 visit.

At this consultation, current medication was recorded, including analgesics in the past 24 hours. Any toxicity from radiotherapy was documented and the questionnaires were repeated. Current symptoms were documented following the week 1 visit, patients received weekly phone calls in order to monitor symptoms and assess analgesic requirements. Week 5 visit: Patients were seen 35 $(+/-5)$ days from the start of radiotherapy. At this visit, all the study visits performed at week 1 were repeated. In addition. However, if patients were unable to attend, efforts were made to see them at home. After the week 5 visit, the weekly phone calls continued until the week 12 visit Week 12 visit: At the week 12 visit, all assessments

Undertaken at week 5 were repeated. Following this visit, patients were discharged back to their local oncology teams and study involvement ceased. During the course of the study, if patients' analgesia required to be altered, this was done as per usual clinical practice.

\section{Questionnaires: Brief Pain Inventory (BPI)}

The BPI is a multi-dimensional pain assessment tool. It was designed to serve two purposes; to measure the intensity of pain and to assess the level of interference of pain on daily function. It was developed for use in cancer patients and has been extensively validated in both cancer and non-cancer patients [3,4] All questions in the BPI relate to the previous 24 hours. The section on pain intensity asks the worst, least and average pain as well as the pain right now. Subjects are asked to score each answer from 0-10 where 0 is "no pain" and 10 is "pain as bad as you can imagine". It also asks the participant to rate the percentage of pain relief they experience from whatever pain treatments or medications they are currently on, ranging from $0-100$. The second section of the BPI focuses on the level of interference of pain on the subject's lifestyle, namely their general activity, mood, walking ability, normal work, relations with other people, sleep and enjoyment of life. Again, the scores are from 0-10 with 0 corresponding to "does not interfere" and 10 representing "completely interferes" with each question that has been asked. Once the questionnaire has been completed, the total BPI score can be calculated and repeated to assess the impact of an intervention on the subject's pain. For the study, the total score at baseline was calculated. A pain response was taken as a30\% drop in BPI score from the baseline assessment [4].

\section{Short Form Mc Gill Pain Question (SF-MPQ)}

The MPG is a scale for assessing pain using verbal descriptors. It was designed to allow patients to express the intensity and quality of their pain [5]. A short form version was developed in 1987 [6]. In 2009 , this was further modified in order to develop a single measure for both neuropathic and non-neuropathic pain. [7] Amongst other purposes, it was planned that this questionnaire, SF-MPQ2, could be used in treatment response studies lead Assessment of Neuropathic symptoms and sign (LANSS)The LANSS was developed in 2001 as a tool to identify patients who are likely to have neuropathic pain [8]. It has been extensively validated $[9,10]$. The assessment consists of two sections; a pain questionnaire and sensory testing. In the pain 
questionnaire, subjects are asked five yes/no questions concerning their pain. With the sensory testing, the subject is examined for alloying and for altered pin-prick threshold. Combining the scores for the questionnaire and the sensory testing gives a maximum

Table 4:

\begin{tabular}{|c|c|c|}
\hline Symptoms & Patient group & Average low SIR \\
\hline Partial bone pain relief & B & 70 \\
\hline Complete bone pain Relief & B & 28 \\
\hline Relief chest pain & D & 59 \\
\hline Brain metastatic & $\mathrm{C}$ & 50 \\
\hline Spinal cord compression & A & 64 \\
\hline
\end{tabular}

QLQ-C30 is a validated questionnaire designed to assess the quality of life of cancer patients [10]. It incorporates nine multi-item scales: five functional scales (physical, role, cognitive, emotional and social); three symptom scales (fatigue, pain, and nausea and vomiting); and a global health and quality-of-life scale. As can be seen in the paragraphs above, with the exception of the NRS for night sweats, all of the questionnaires used in the study have been validated. The first step in the validation process involves a questionnaire being reposed as a potential way of measuring a symptom, e.g. pain. The validation comes when the questionnaire is tested on a different set of patients and similar results are obtained (Table 5).

Table 5:

\begin{tabular}{|c|c|c|}
\hline Metastic site & Code & Frequency \\
\hline Spinal compression & A & 38 \\
\hline Bone Metastic & B & 25 \\
\hline Brain metes & C & 18 \\
\hline
\end{tabular}

\section{The Results}

Table 6: Shows cancer Metastic site for patients' evaluation in this study.

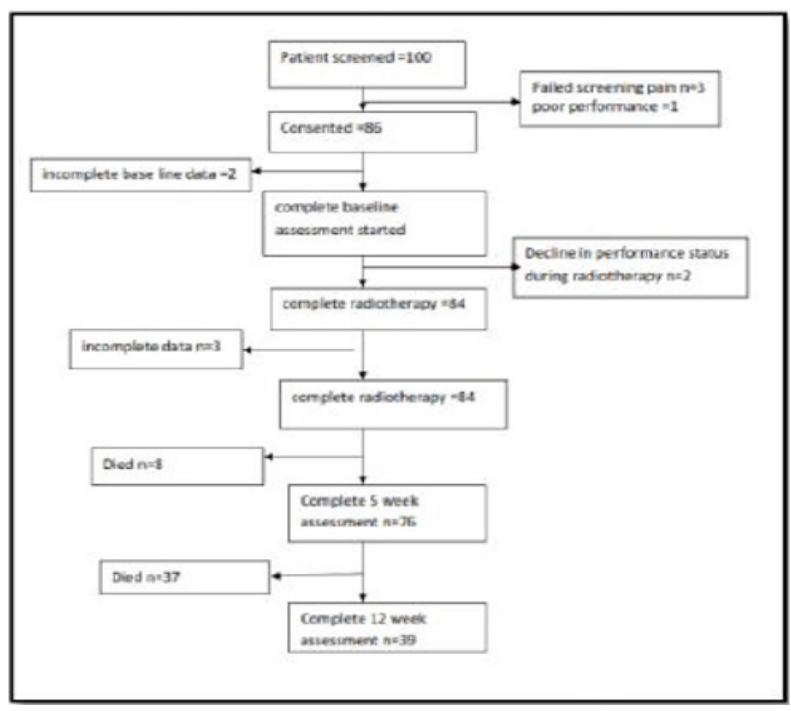

score of 24 . A score of $>12$ suggests that neuropathic mechanisms are likely to be contributing to the patient's pain, whereas a score of $<12$ suggests that neuropathic mechanisms are unlikely to be contributing to the patient's pain (Table 4).

\begin{tabular}{|c|c|c|}
\hline High point in base line & Low point in base line & Average high SIR \\
\hline 100 & 0 & 90 \\
\hline 100 & 0 & 80 \\
\hline 100 & 0 & 86 \\
\hline 100 & 0 & 70 \\
\hline 100 & 0 & 73 \\
\hline
\end{tabular}

Thirty-nine patients returned completed questionnaires (of 100 expected, deceased patients excluded) during the initia12 weeks study period (Table 6) (Figure 2).

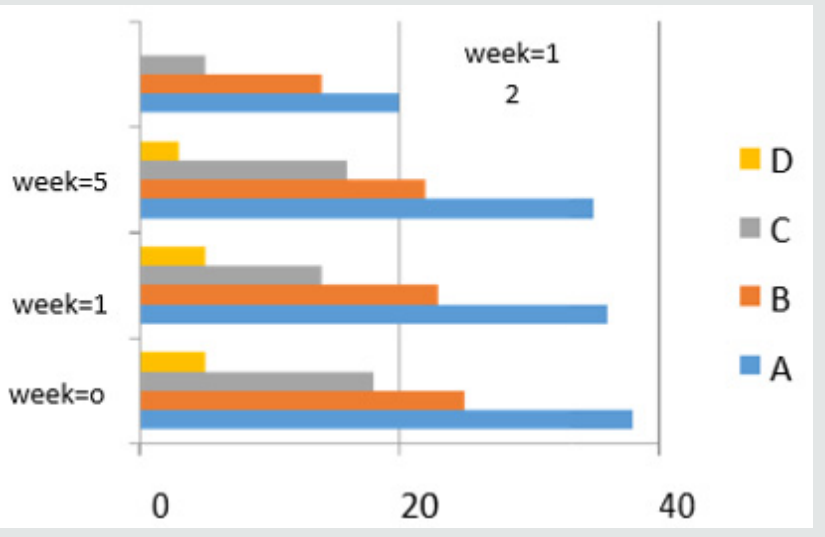

Figure 2: Show the survival patients during study period $(\mathrm{A}=$ spinal cord compression, $\mathrm{B}=$ bone metes, $\mathrm{C}=$ brain metes, $\mathrm{D}=$ lung metes).

\section{Symptoms Improvement Ratio (SIR)}

This concept corresponds with Metastic site and symptoms related to it, the table below show in group A there were high rate symptoms improvement, but it can be change in group $\mathrm{B}$ and $\mathrm{C}$ (Figures 3 \& 4).

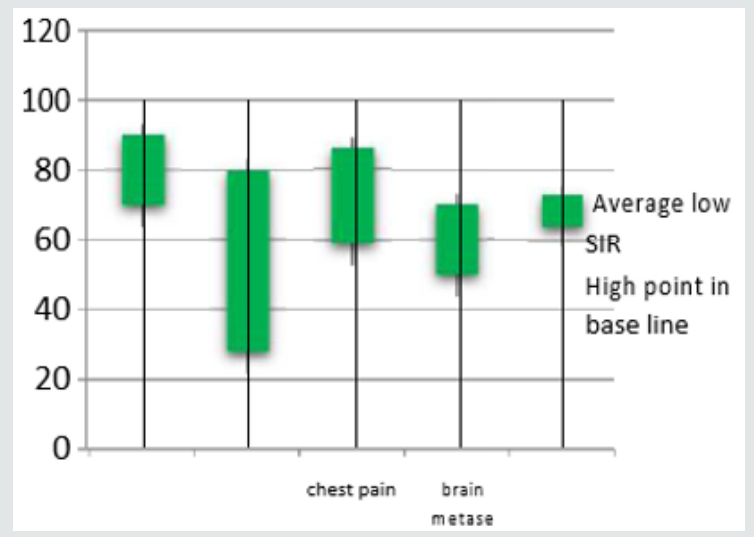

Figure 3: Symptoms improvement ratio according to patient group. 


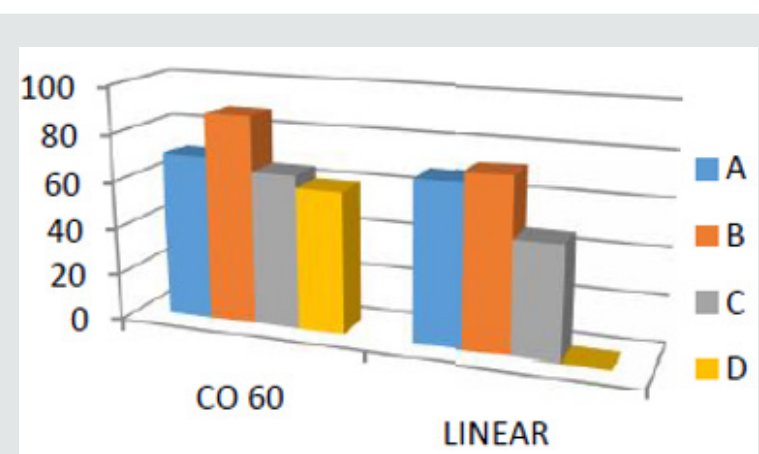

Figure 4: Cross tabulation between SIR and Treatment Machine Modality.

\section{Conclusion}

In metastatic breast cancer patients suffer from many symptoms related with site of metastasis such as pain, dyspnea, bleeding, problem with vision .... etc., this symptoms effect in patient's daily life by decrease the quality of life and increase pain and toxicity Some time the chemotherapy can't control the localized pain or compression, also the oncologist didn't prefer to be used it because the toxicity will be increase and the few responses. Palliative radiotherapy plays important role in management of this symptoms, symptoms improvement ratio will help all to evaluation the benefit of radiotherapy in palliative cases. A major goal of palliative radiotherapy is controlling the symptoms by given the tumour dose the lethal dose with simple treatment planning and treatment. However, patient's symptoms such as pain, compression and brain Metastic symptoms can be controlled by radiotherapy. The study was conducted at different oncology hospitals in Sudan (Khartoum oncology hospital (RICK), for specific purpose of acquiring date for study. The study was conducted from 2014 to 2017.Symptoms improvement ratio (SIR) has been develop and become more and more popular in cancer management team. The SIR represents, in absolute terms, the proportion of patients who, following treatment, have a clearly documented improvement in one or more predetermined objective and evaluable symptoms. It represents the average or approximate rate of clinically significant improvement in a specific symptom such as pain.

\section{Recommendations}

The researcher recommended that develop of Palliative Radiotherapy program base use Symptoms improvement ratio concept as reference concept to evaluation the role of radiotherapy in controlled the symptoms in palliative cases when deliver radiotherapy for patients in late stage breast cancer as palliative treatment many factors should be consider:

a. Implantation of stander palliative radiotherapy program in each oncology hospital play important role in success the RT treatment.

b. The time is important factor which effect in patient's response, time between the symptoms appearance and treat with first session.

c. The radiotherapy regime which describe the suitable field size with minimum number of fractions should be chosen.

d. Fellow up the patient should be consider in all palliative case to manage any incident before it appears.

\section{Acknowledgment}

I am most of all grateful to the God, who made me able to fulfil this $\mathrm{PhD}$ thesis in spite of all problem and pressure. Profound thanks and gratitude to everyone who encouraged me to complete this thesis. I named my thesis to all breast cancer patients fighting and win, my parents, my husband and my kids whose prayers and support has always contributed to my success. My supervisor professor Mohamed Alfadil deserves all my gratitude as I could not have made it through without his invaluable support.

\section{References}

1. Jemal A, Bray F, Center MM, Ferlay J, Ward E, et al. (2011) Global cancer statistics. CA: A Cancer Journal for Clinicians 61(2): 69-90.

2. Cardoso F, Harbeck N, Fallowfield L, Kyriakides S, Senkus E, et al. (2012) Locally recurrent or metastatic breast cancer: ESMO Clinical Practice Guidelines for diagnosis, treatment and follow-up. Annals of Oncology 23(Suppl 7): 11-19.

3. Cleeland CS, Ryan KM (1994) Pain assessment: global use of the Brief Pain Inventory. Annals Academy of Medicine Singapore 23(2): 129-138.

4. Portenoy RK, Payne D, Jacobsen P (1999) Breakthrough pain: characteristics and impact in patients with cancer pain. Department of Pain Medicine and Palliative Care 81(1-2): 129-134.

5. Melzack R (1975) The McGill Pain Questionnaire: major properties and scoring methods. Pain 1(3): 277-299.

6. Melzack R (1987) The short-form McGill Pain Questionnaire. Pain 30(2): 191-197.

7. Dworkin RH, Turk DC, Revicki DA, Harding G, Coyne KS, et al. (2009) Development and initial validation of an expanded and revised version of the Short-form McGill Pain Questionnaire (SF-MPQ-2). Pain 144(1-2): 35-42.

8. Bennett M (2001) The LANSS Pain Scale: the Leeds assessment of neuropathic symptoms and signs. Pain 92(1-2): 147-157.

9. Kaki AM, El Yaski AZ, Youseif E (2005) Identifying neuropathic pain among patients with chronic low-back pain: use of the Leeds Assessment of Neuropathic Symptoms and Signs pain scale. Regional Anesthesia and Pain Medicine 30(5): 422-428.

10. Aaronson NK, Ahmedzai S, Bergman B, Bullinger M, Cull A, et al. (1993) The European Organization for Research and Treatment of Cancer QLQ-C30: a quality-of-life instrument for use in international clinical trials in oncology. Journal of the National Cancer Institute 85(5): 365376. 
(C) (i) This work is licensed under Creative Commons Attribution 4.0 License

To Submit Your Article Click Here: Submit Article

DOI: $10.32474 /$ OAJOM.2019.02.000148

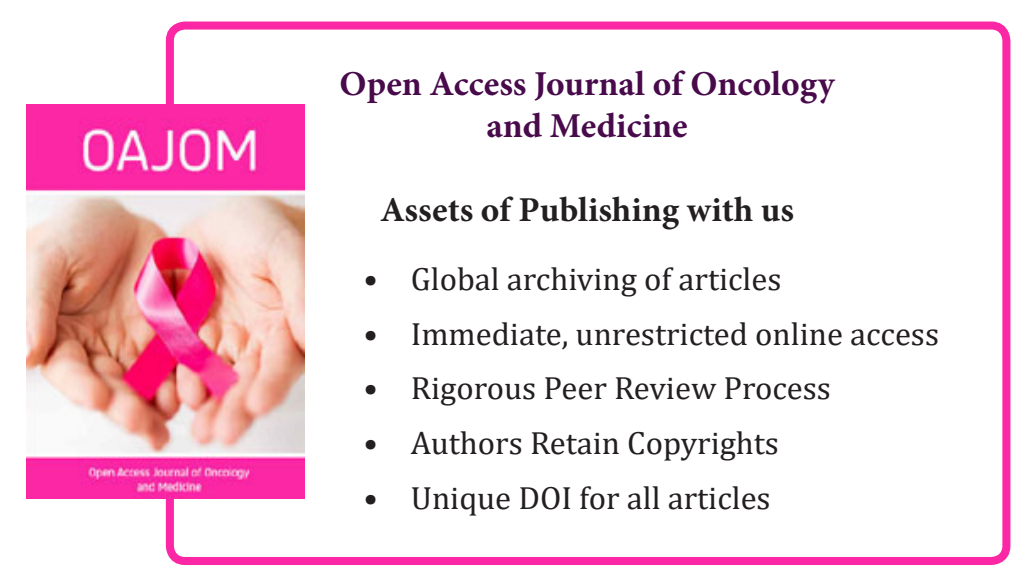

